\title{
Proliferation inhibition and differentiation induction of hepatic cancer stem cells by knockdown of BC047440: A potential therapeutic target of stem cell treatment for hepatocellular carcinoma
}

\author{
NAN YOU ${ }^{1 *}$, LU ZHENG $^{1 *}$, WEIHUI LIU ${ }^{2 *}$, XIAO ZHONG ${ }^{3}$, WEIWEI WANG ${ }^{1}$ and JING LI ${ }^{1}$ \\ ${ }^{1}$ Department of Hepatobiliary Surgery, Xinqiao Hospital, Third Military Medical University, Chongqing 400037; \\ ${ }^{2}$ PLA Center of General Surgery, General Hospital of Chengdu Army Region, Chengdu, Sichuan 610083; \\ ${ }^{3}$ Department of Urology, Xinqiao Hospital, Third Military Medical University, Chongqing 400037, P.R. China
}

Received January 7, 2014; Accepted February 10, 2014

DOI: $10.3892 /$ or.2014.3043

\begin{abstract}
Recent findings suggest that clinical hepatocellular carcinoma (HCC) progression is driven by hepatic cancer stem cells (HCSCs) through their capacity for self-renewal, generation of heterogeneous lineages of cancer cells, resistance to chemotherapy and their ability to divide limitlessly, which may contribute to the failure of existing therapies to consistently eradicate malignant tumors. Therefore, HCSCdirected therapeutic approaches might represent strategies to improve clinical HCC therapy. In previous studies, we showed that $\mathrm{BC} 047440$ was found to play a critical role in mediating HCC cell proliferation. The present study sought to determine whether BC047440 is involved in maintaining HCSC malig-
\end{abstract}

Correspondence to: Professor Jing Li, Department of Hepatobiliary Surgery, Xinqiao Hospital, Third Military Medical University, Chongqing 400037, P.R. China

E-mail: jinglixq@yeah.net

${ }^{*}$ Contributed equally

Abbreviations: AFP, $\alpha$-fetoprotein; ALB, albumin; BSA, bovine serum albumin; CSCs, cancer stem cells; CYP1A2, cytochrome P450 1A2; DMEM, Dulbecco's modified Eagle's medium; EDTA, ethylenediaminetetraacetic acid; ELISA, enzyme-linked immunosorbent assay; EST, expressed sequence tag; FBS, fetal bovine serum; FITC; fluorescein isothiocyanate; GAPDH, glyceraldehyde-3-phosphate dehydrogenase; HCC, hepatocellular carcinoma; HCSCs, hepatic cancer stem cells; HGF, hepatocyte growth factor; HNF $4 \alpha$, hepatocyte nuclear factor $4 \alpha$; HNSCs, hepatic normal stem cells; HRP, horseradish peroxidase; LSD, least significant difference; $\mathrm{NC}$, negative control; $\mathrm{NF}-\kappa \mathrm{B}$, nuclear factor- $\kappa \mathrm{B}$; PBS, phosphate-buffered saline; RACE, rapid amplification of cDNA ends; shRNA, short hairpin RNA; TEM, transmission electron microscopy; $\mathrm{V}$, volume

Key words: BC047440, hepatocellular carcinoma, hepatic cancer stem cells, targeted therapy nant behavior (including proliferation and differentiation). We demonstrated that $\mathrm{BC} 047440$ expression was markedly upregulated in HCSCs. Furthermore, we inhibited BC047440 in HCSCs using short hairpin RNA (shRNA). The effects of BC047440 on proliferation and differentiation were investigated. We also analyzed the involvement of critical molecular events known to regulate the proliferation and the differentiation machinery. Excluding apoptosis-related effects, we found that BC047440 inhibition resulted in enhanced cell proliferation through enhancing cytoplasmic accumulation of nuclear factor- $\mathrm{\kappa B}(\mathrm{NF}-\mathrm{\kappa B})$ with a concomitant decrease in the nuclear fraction. BC047440 inhibition also resulted in inducing HCSC differentiation into hepatocytes. Furthermore, following downregulation of $\mathrm{BC} 047440$, the level of hepatocyte nuclear factor $4 \alpha(\mathrm{HNF} 4 \alpha)$ increased. Finally, tumorigenicity suppression following BC047440 depletion was confirmed in a nude mouse model. In conclusion, our findings indicate that BC047440 plays an important role in the proliferation and differentiation of HCSCs and may represent a novel therapeutic target for the treatment of $\mathrm{HCC}$.

\section{Introduction}

In-depth studies have demonstrated that tumor tissues are composed of cells at different stages of differentiation, including a subset of cells termed cancer stem cells (CSCs) that have the potential for self-renewal and differentiation. These tumor-initiating cells are involved in tumorigenesis, development, metastasis, recurrence and drug resistance. A determining role of CSCs in tumorigenesis has been recognized in breast and hematological cancers, as well as in nervous system tumors $(1,2)$. The continuous development of the CSC theory has brought new ideas to tumorigenesis research and novel methods for diagnosing and treating cancer.

Hepatocellular carcinoma (HCC) is among the most aggressive human diseases, and its pathogenesis remains unclear. There is extensive evidence that HCC is a stem cellderived disease. Hepatic cancer stem cells (HCSCs) are the initiating cells of $\mathrm{HCC}$ and are important in maintaining HCC 
growth, metastasis and recurrence, whereas non-stem cell-like HCC cells do not have self-renewal capacity and ultimately die after limited proliferation (3). The novel CSC theory could have a significant impact on the traditional HCC treatment strategy. Currently, all HCC cells are targeted by surgical treatment, radiotherapy and/or chemotherapy. Although eliminating the vast majority of $\mathrm{HCC}$ cells with limited proliferative capacity can promote tumor regression, it cannot eradicate the tumor source (4). Compared with non-stem cell-like HCC cells, HCSCs have high proliferative capacity and tumorigenicity and can effectively initiate the DNA SOS response and increase the expression of drug resistance-related proteins that facilitate chemotherapy resistance and allow tumor cells to survive treatment, which ultimately results in HCC recurrence. Therefore, it is necessary to effectively eradicate HCSCs to achieve therapeutic efficacy against HCC. This may be possible if specific stem cell signals are inhibited using gene therapy, while at the same time attacking proliferating cells by conventional therapy. However, key genes and related pathways in HCSC research are still preliminary $(5,6)$.

With support from the National Natural Science Foundation of China, in the present study we isolated an expressed sequence tag (EST) fragment that was highly expressed in HCC tissue using the suppression subtractive hybridization technique and then obtained a full-length cDNA sequence (1476 bp) using the rapid amplification of cDNA ends (RACE) method. A GeneBank search revealed that it was identical to a recently reported novel gene with unknown function (GeneBank accession number: BC047440). The gene is located on 20q11.22. PROSITE analysis showed that this gene might contain three protein kinase $\mathrm{C}$ phosphorylation sites, two casein kinase II phosphorylation sites, two myristyl sites and one asparagine-linked glycosylation site. The subcellular localization of the protein is likely cytosolic. The BC047440encoded protein is predicted to be composed of 200 amino acids, with an isoelectric point of 6.32 and a molecular weight of $22.6 \mathrm{kDa}$. Our previous studies suggested that the novel HCC-related gene BC047440 is associated with HCC development and progression. Furthermore, evidence suggests that BC047440 plays a crucial role in mediating HCC proliferation and differentiation (Fig. 1) of HCC cells (7-9). Nevertheless, few studies have assessed the role of BC047440 in HCSCs. Based on our previous findings regarding the determining role of HCSCs in tumorigenesis, we hypothesized that BC047440 might be involved in maintaining HCSC malignant behavior (including proliferation and differentiation) and might be useful for gene-targeted therapies.

In order to further elucidate the specific mode of BC047440 action, in the present study, we investigated the effect of BC047440 inhibition on HCSC proliferation and differentiation. We also elucidated the molecular mechanisms that underlie these processes. To the best of our knowledge, this is the first report describing the effects of BC047440 inhibition in HCSCs. Loss of BC047440 gene expression results in the proliferation inhibition of HCSCs with concomitant differentiation, eventually leading to tumorigenicity suppression. The results of the present research support a novel role of BC047440 in hepatic tumorigenesis and will help revolutionize therapeutic strategies and provide unique opportunities for HCC gene therapy.

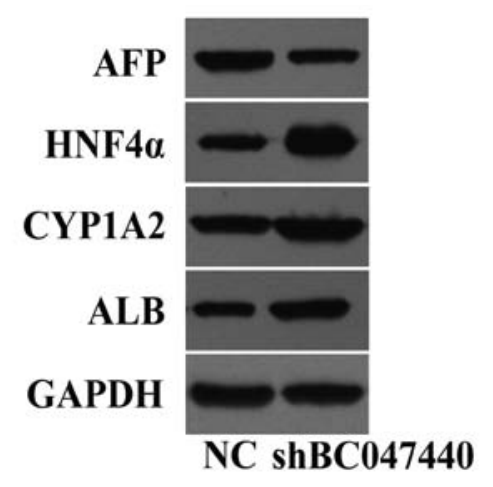

Figure 1. Western blot analysis shows the effects of downregulated BC047440 on the expression of AFP, ALB, HNF4 $\alpha$ and CYP1A2 in HepG2 cells.

\section{Materials and methods}

Animals. Fischer 344 rats (250-300 g body weight) were purchased from the Shanghai Slack Experimental Animal Center, China. Nude mice were purchased from the Laboratory Animal Center of Beijing Xiehe Medical University, China. All rats were housed under standardized conditions [room at constant temperature $\left(25^{\circ} \mathrm{C}\right)$ with alternating 12 -h periods of light and darkness] and fed a standard rat diet with free access to water. Food and water were given ad libitum. All experimental procedures involving animals were approved by the Local Animal Care and Use Committee of the Third Military Medical University.

Isolation of hepatic normal stem cells (HNSCs) and HCSCs and cell culture. HNSCs and HCSCs were respectively obtained by procedures previously reported by our group $(10,11)$. Cells were grown in Williams' E medium (AppliChem GmbH, Darmstadt, Germany) supplemented with $10 \%$ fetal bovine serum (FBS; Gibco/Invitrogen, Grand Island, NY, USA), $100 \mathrm{IU} / \mathrm{ml}$ penicillin, $400 \mathrm{IU} / \mathrm{l}$ trypsin and $100 \mu \mathrm{g} / \mathrm{ml}$ streptomycin. The cells were plated in $75-\mathrm{cm}^{2}$ flasks and cultured at $37^{\circ} \mathrm{C}$ with $5 \% \mathrm{CO}_{2}$ and $95 \%$ humidified air. The medium was changed every 2 days.

Analysis of the BC047440 gene expression between HNSCs and HCSCs. HNSCs and HCSCs were processed for protein extraction, and western blot analysis was performed according to the published method. The primary antibodies used were: anti-BC047440 (diluted 1:500, self-prepared) and anti-glyceraldehyde-3-phosphate dehydrogenase (GAPDH; diluted 1:400, mouse monoclonal C-2; Santa Cruz Biotechnology, Santa Cruz, CA, USA).

short hairpin RNA (shRNA) lentiviral infection of HCSCs. HCSCs were plated at $2 \times 10^{5}$ cells/well in $1 \mathrm{ml}$ of Williams' E supplemented with $10 \%$ FBS in a 12-multiwell plate. Infection was performed at a cell confluency of $\sim 50-70 \%$ using commercial shRNA lentiviral particles (Shanghai GeneChem Co., Ltd., Shanghai, China) according to the manufacturer's protocol. An invalid RNAi sequence was used as a negative control (NC). Puromycin was used to screen stably transfected clones. Established stable cell lines, HCSCs (shBC047440) 
or HCSCs (NC), were then cultured in medium containing $0.5 \mu \mathrm{g} / \mathrm{ml}$ puromycin. Knockdown of $\mathrm{BC} 047440$ was verified by western blot analysis for successful silencing.

\section{The effects of BC047440 on HCSC proliferation}

Annexin V/propidium iodide (PI) assay. To exclude apoptosis-related effects, Annexin V assays were performed using an apoptosis detection kit [Annexin V-fluorescein isothiocyanate (FITC)/PI Staining kit; Immunotech Co., Marseille, France] as described in the manufacturer's instructions. Briefly, the different interfered cells were collected, washed in cold phosphate-buffered saline (PBS), incubated for 15 min with a fluorescein-conjugated Annexin V and PI and analyzed using flow cytometry (Becton-Dickinson, San Jose, CA, USA).

Cell counting. HCSCs (shBC047440) and HCSCs (NC) were digested with $0.25 \%$ trypsin and $0.01 \%$ ethylenediaminetetraacetic acid (EDTA), adjusted to a density of $1 \times 10^{4}$ cells $/ \mathrm{ml}$

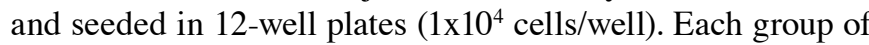
cells included 3 parallel samples. Every day during a period of 7 days, the cells of the 4 parallel samples in each group were trypsinized and the cell number was counted under an inverted microscope (Olympus, Tokyo, Japan).

Colony formation assay. Approximately $3 \times 10^{2}$ cells from the different interfered cells were plated in six-well dishes. After 7 days, cells were fixed with $20 \%$ methanol and stained with $1 \%$ crystal violet. Colonies consisting of $>50$ cells were counted per well, and each experiment was performed in triplicate.

Analyses of the expression of the cellular proliferation marker $\mathrm{Ki}$-67. Flow cytometric analysis was performed for the Ki-67 studies. Briefly, the different interfered cells were harvested, fixed in $70 \%(\mathrm{v} / \mathrm{v})$ ethanol, washed and incubated for $20 \mathrm{~min}$ with $1 \%$ bovine serum albumin (BSA). The cells were then incubated with 1:200 diluted anti-Ki-67 (FITC conjugated; Bioscience, Beijing, China). The data were obtained and analyzed using flow cytometry.

Cell cycle distribution analysis. Cell cycle analysis assays are used in flow cytometry. HCSCs (shBC047440) and HCSCs (NC) were fixed in ice-cold $70 \%(\mathrm{v} / \mathrm{v})$ ethanol for $48 \mathrm{~h}$ at $4^{\circ} \mathrm{C}$. They were then washed once with PBS and centrifuged at $200 \times \mathrm{g}$ for $10 \mathrm{~min}$, followed by treatment with RNase A at $1 \mathrm{mg} / \mathrm{ml}$ for $30 \mathrm{~min}$ at $37^{\circ} \mathrm{C}$. After staining with $40 \mu \mathrm{l}$ of $0.1 \mathrm{mg} / \mathrm{l} \mathrm{PI}$, histograms of DNA content were analyzed using flow cytometry to determine cell cycle distribution $(\mathrm{G} 0 / \mathrm{G} 1, \mathrm{~S}$, and $\mathrm{G} 2 / \mathrm{M}$ phase).

Western blot analysis for the effects of BC047440 on the activation of nuclear nuclear factor- $\kappa B(N F-\kappa B)$ in HCSCs. The preparation of cytoplasmic and nuclear extracts was performed using the Nuclear Extract kit (HyClone-Pierce, Logan, UT, USA) according to the manufacturer's instructions. To measure nuclear NF- $\kappa \mathrm{B} / \mathrm{p} 65$ and cytoplasmic NF- $\kappa \mathrm{B} /$ p65 expression, western blot analysis was performed. Related fusion proteins were identified using anti-NF- $\kappa \mathrm{B} / \mathrm{p} 65$ primary antibody (Santa Cruz Biotechnology).
The effects of BC047440 on HCSC differentiation

Detection of stem cell markers by flow cytometric analysis. The stem-cell-associated markers of different interfered cells were analyzed by flow cytometric analysis. Briefly, at 7 days of HCSC (shBC047440) and HCSC (NC) growth in the presence of hepatocyte growth factor (HGF), the cells were prepared as single cell suspensions at a density of $1 \times 10^{6}$ cells $/ \mathrm{ml}$ using Dulbecco's modified Eagle's medium (DMEM) (containing $20 \%$ FBS) and incubated for 15-30 min at room temperature to block non-specific sites. These cells were then washed twice with PBS and re-suspended in $990 \mu \mathrm{l}$ PBS. Subsequently, $10 \mu 1$ of antibodies, including CD133 (PE-conjugated; BioLegend, San Diego, CA, USA) and EpCAM (FITC-conjugated, BioLegend), were added to each cell suspension. After $30 \mathrm{~min}$ of incubation at $4^{\circ} \mathrm{C}$ in the dark, the cells were washed twice with PBS, fixed in $0.1 \%$ formaldehyde and analyzed using flow cytometry.

Measurement of albumin (ALB), $\alpha$-fetoprotein (AFP) and urea levels. The conditioned media from HCSCs (shBC047440) and HCSCs (NC) cultured with HGF were collected at day 7 and were used for assaying AFP and ALB production with enzyme-linked immunosorbent assay (ELISA) kits (Cusabio Biotech Co., Ltd., Wuhan, China) according to the manufacturer's protocol. The cells from each condition were incubated with $\mathrm{NH}_{4} \mathrm{Cl}(5 \mathrm{mM} / \mathrm{ml}$; Sigma, St. Louis, MO, USA) for $24 \mathrm{~h}$ in $5 \% \mathrm{CO}_{2}$ at $37^{\circ} \mathrm{C}$ on day 7 . Following incubation, the supernatants were collected and the urea concentrations were measured using a colorimetric assay kit (Gdb Corp., San Diego, CA, USA).

Transmission electron microscopy (TEM). Morphological changes in HCSCs (shBC047440) and HCSCs (NC) treated with HGF were evaluated by TEM. After incubation with HGF for 7 days, the cells were digested with trypsin and fixed in $2.5 \%$ glutaraldehyde precooled at $4^{\circ} \mathrm{C}$ for $2 \mathrm{~h}$. To make ultra-thin sections for copper staining, cells were washed with PBS, fixed in $1 \%$ osmic acid for an additional hour, dehydrated in acetone and embedded in epoxide resin. After the sections were stained with uranyl acetate and lead citrate, the ultrastructural features of cells were observed under an electron microscope (JEM-2000EX; JEOL Ltd., Tokyo, Japan).

Western blot analysis for the effects of BC047440 on hepatocyte nuclear factor $4 \alpha(H N F 4 \alpha)$ expression. HNF $4 \alpha$ expression was measured by western blot analysis. Western blot analysis was performed using techniques previously described. Briefly, HNF4 $\alpha$ fusion protein was identified using anti-HNF4 $\alpha$ primary antibody (diluted 1:500, rabbit polyclonal; Santa Cruz Biotechnology) and the horseradish peroxidase (HRP)-conjugated anti-rabbit IgG (diluted 1:2,000) as secondary antibody.

Tumorigenesis assay. To determine the effects of BC047440 knockdown on the tumorigenic potential of HCSCs in vivo, we compared the growth of tumors produced by injection of $2 \times 10^{6}$ HCSCs (shBC047440) on the left side of the back with tumors produced by injection of $2 \times 10^{6}$ HCSCs (NC) on the right side of the same animal's back. Tumor size was 
A

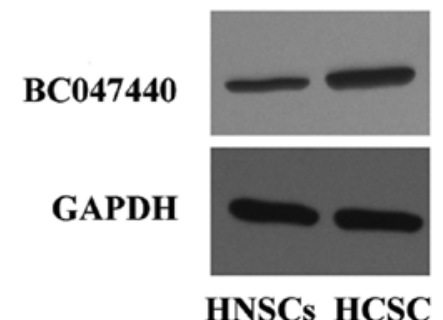

C

BC047440

GAPDH
HNSCs HCSC

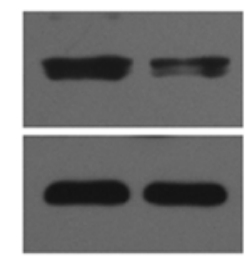

NC shBC047440
B

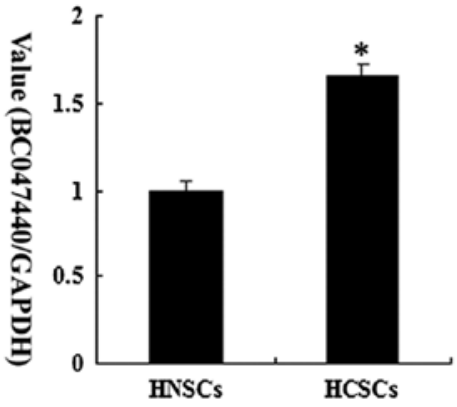

D

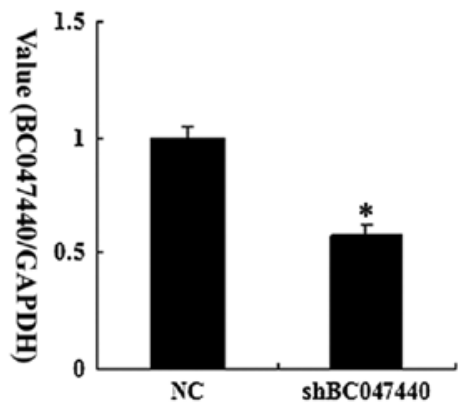

Figure 2. (A) Detection of BC047440 protein expression in HNSCs and HCSCs as determined by western blot analysis. GAPDH was used as a loading control. (B) Least significant difference (LSD) tests by ANOVA were performed to determine the differences in the BC047440/GAPDH ratio between groups. ${ }^{\text {"P }}<0.05$ compared to HNSCs group. (C) Western blot analysis of the inhibition of BC047440 protein in the different interfered cells. The different interfered cells were lysed and subjected to immunoblot analysis. (D) LSD tests by ANOVA were performed to determine the differences in the BC047440/GAPDH ratio between groups. ${ }^{\text {P }}<0.05$ compared to control group.

A

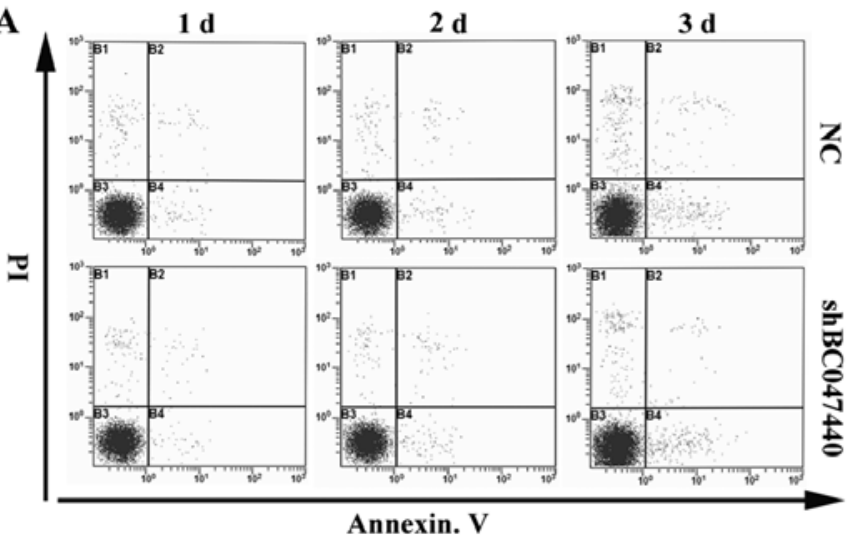

B

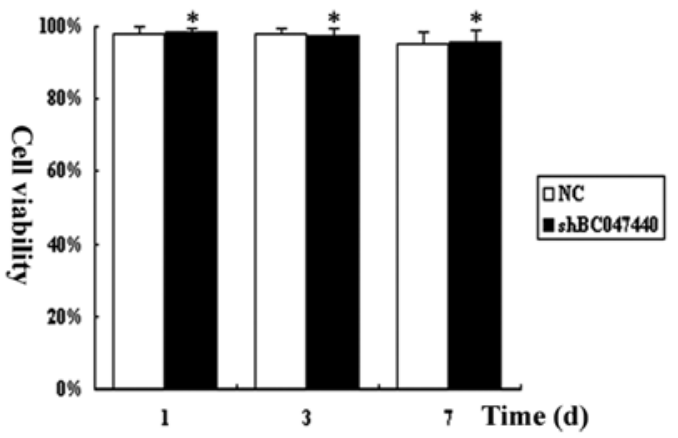

Figure 3. (A) To exclude the effects of BC047440 on cell viability, Annexin V assays were performed. Culture cells were treated for the indicated time periods and then stained with FITC-conjugated Annexin V and PI. (B) The percentage of viable cells in each group is reflected in the column chart. "P>0.05 compared to control group.

measured twice weekly. Tumor volume (V) was calculated using the following formula: $\mathrm{V}=(\mathrm{a} \times \mathrm{b} \times \mathrm{c}) / 2$, where $\mathrm{a}$ and $\mathrm{b}$ are the shorter and longer diameters of each tumor, respectively, and $\mathrm{c}$ is the thickness. The animals were sacrificed after 40 days.

Statistical analysis. The significance of differences between the groups was determined with one-way ANOVA (SPSS 10.0 statistical software). The results with a P-value $\leq 0.05$ were considered statistically significant. Data are expressed as the mean \pm the standard error of the mean of separate experiments $(n \geq 3$, where $n$ represents the number of independent experiments).

\section{Results}

Isolation of HNSCs and HCSCs. According to the special density characteristic of stem cells, we have enriched special density HNSCs and HCSCs via a density gradient centrifugation-centered method $(10,11)$. Our previous data revealed that the derived cells exhibit stem cell-like characteristics, including high tumorigenicity.

Increased BC047440 expression in HCSCs. BC047440 expression was compared between HCSCs and HNSCs by western blot analysis. The levels of BC047440 expression in HCSCs were higher than those for HNSCs (Fig. 2A and B). 


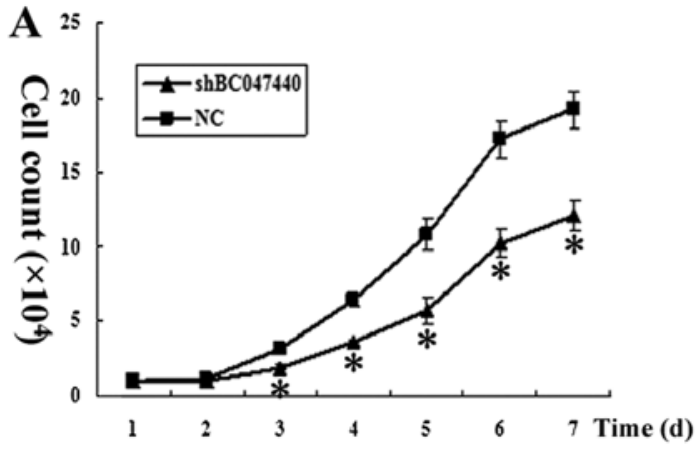

C

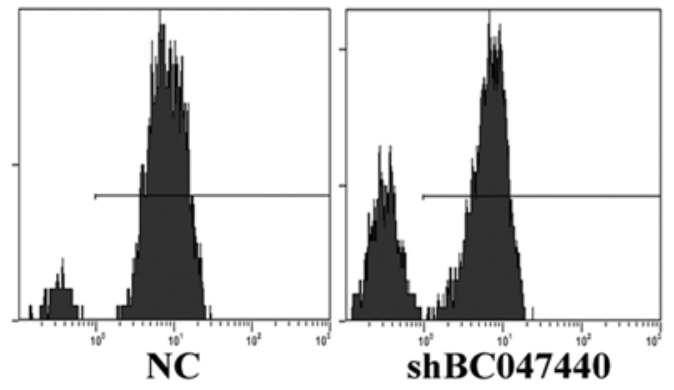

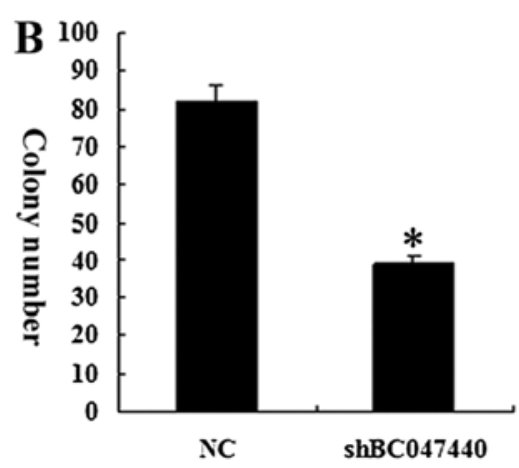

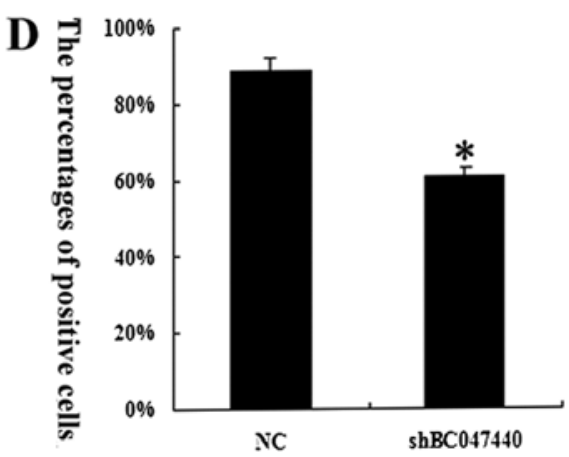

Figure 4. Silencing of the BC047440 gene resulted in decreased cell number, as determined by (A) cell counting and (B) colony formation; the count number of the colonies is shown in the diagram. (C) The inhibition of the BC047440 gene resulted in decreased growth of HCSCs determined by Ki-67 analysis. (D) The expression of $\mathrm{Ki}-67$ in different interfered cells is reflected in the column chart. ${ }^{*} \mathrm{P}<0.05$ compared to control group.

shRNA-mediated downregulation of BC047440 expression. To confirm the silencing of BC047440 expression in HCSCs, western blot analysis was performed. Western blot analysis after shRNA treatment showed a marked decrease in BC047440 protein in HCSCs (Fig. 2C and D). These data show that the lentiviral-mediated shRNA used in the present study was effective in silencing BC047440 gene expression in HCSCs.

Effects of BC047440 on HCSC proliferation. To determine whether BC047440 effects HCSC proliferation, we first examined the effect of BC047440 depletion on cell viability using Annexin V assays. As shown in Fig. 3, over the time course of the experiment, BC047440 depletion did not affect the viability of HCSCs and apoptosis had no effect on cell proliferation. Cell counting showed that BC047440 depletion significantly suppressed the proliferation of HCSCs (Fig. 4A). Colony formation analysis showed that BC047440-depleted HCSCs had a markedly reduced capacity to form colonies compared with the control cells (Fig. 4B). Moreover, cell proliferation was assessed by Ki-67 expression analysis (Fig. 4C and D). The expression of Ki-67 in HCSCs (shBC047440) decreased significantly $(\mathrm{n}=3, \mathrm{P}<0.05)$ compared to HCSCs $(\mathrm{NC})$. The results suggest that the silencing of the $\mathrm{BC} 047440$ gene resulted in the inhibition of HCSC proliferation.

To further investigate the effects of BC047440 on HCSC proliferation, we used flow cytometry to determine the cell cycle distribution of HCSCs (shBC047440) and HCSCs (NC). As shown in Fig. 5A, there was a higher proportion of G0-G1 phase cells $(81.47 \%)$ in HCSCs (shBC047440) compared to HCSCs (NC) (58.09\%). A compensatory decrease in the
$\mathrm{S}(12.48 \%)$ and $\mathrm{G} 2 / \mathrm{M}$ phase $(6.05 \%)$ proportions was also detected as compared with the control in S (26.29\%) and G2/M phases $(15.62 \%)$. The data indicate that the downregulation of BC047440 expression in HCSCs may have a significant effect on cell proliferation.

Since NF- $\mathrm{BB}$ activation controls the expression of a number of genes involved in cell growth and survival through direct and indirect mechanisms, we investigated whether BC047440 knockdown has an impact on $\mathrm{NF}-\kappa \mathrm{B}$ activation in HCSCs. We revealed that $\mathrm{BC} 047440$ knockdown caused an increase in NF- $\kappa \mathrm{B}$ levels in the cytoplasmic fraction of HCSCs with a simultaneous decrease in the nuclear fraction (Fig. 5B and C). The results indicate that $\mathrm{BC} 047440$ knockdown suppresses constitutive activation of NF- $\kappa \mathrm{B}$ in HCSCs.

Effects of BC047440 on HCSC differentiation. To determine the stem properties of cells in different interfered cells, CD133 and EpCAM were used as a probe to quantitatively analyze the profile of stem-like cells in HCSCs (shBC047440) and HCSCs (NC). On day 7 of cell growth in the presence of HGF, CD133 percentages in HCSCs (NC) and HCSCs (shBC047440) were $47.9 \pm 2.5$ and $26.1 \pm 2.1 \%$, respectively. EpCAM percentages in HCSCs (NC) and HCSCs (shBC047440) were 37.1 \pm 1.9 and $21.4 \pm 1.3 \%$, respectively (Fig. 6). These data indicated that BC047440 can modulate the stemness properties of HCSCs.

Stemness properties were also evaluated at the functional level. On day 7 of cells treated with HGF, the production of ALB in HCSCs (shBC047440) was significantly higher than in HCSCs (NC), and an expected decrease in AFP production was observed on day 7 in HCSCs (shBC047440) compared to HCSCs (NC) (Fig. 7A and B). In addition, upon treatment with 

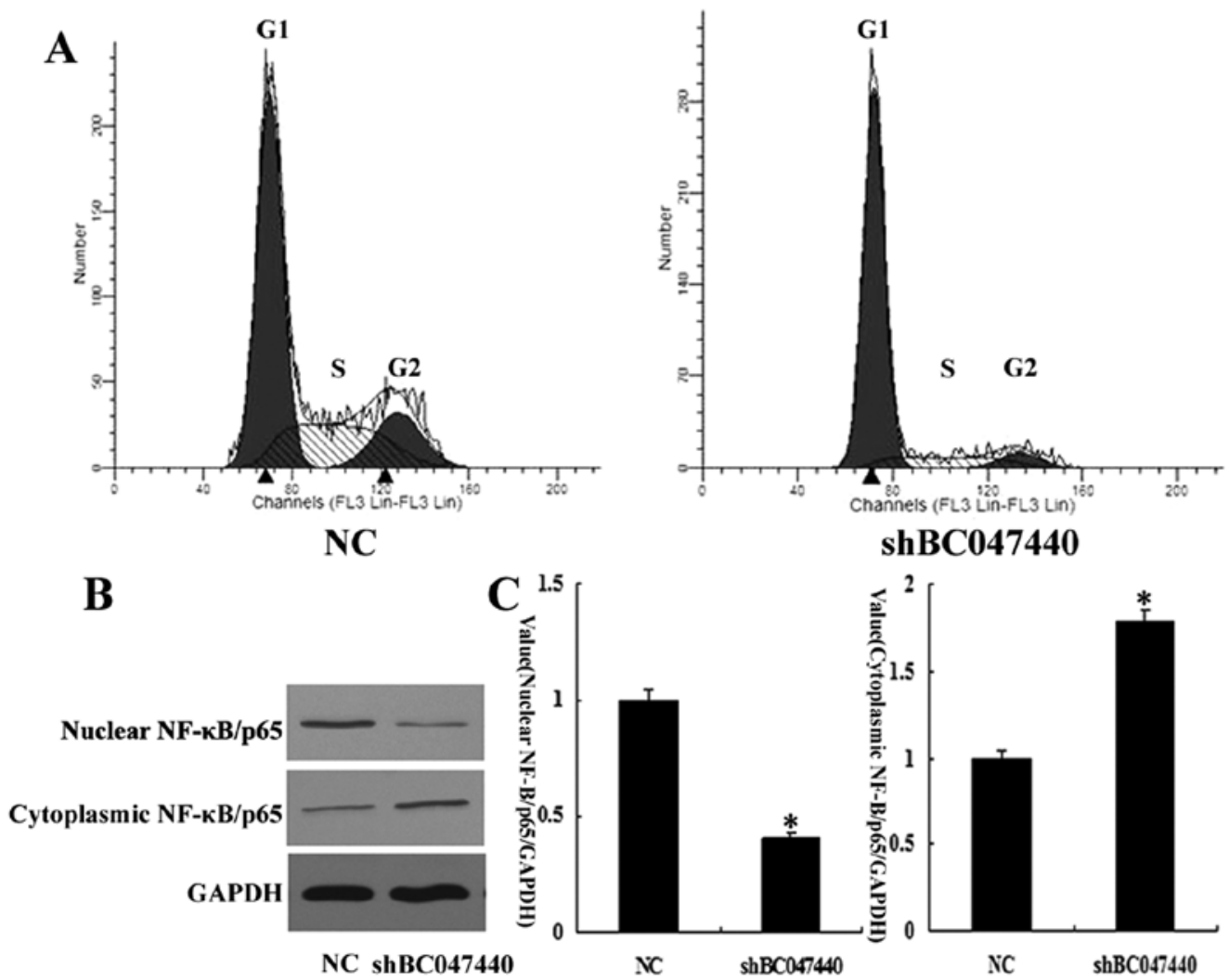

Figure 5. (A) DNA levels in each group. Downregulation of BC047440 significantly increased the number of cells in the G0/G1 of the cell cycle and decreased the percentage of $\mathrm{S}$ and $\mathrm{G} 2 / \mathrm{M}$ phase cells. (B) BC047440 knockdown attenuated constitutive NF- $\kappa \mathrm{B}$ activation by inhibiting nuclear translocation of NF- $\mathrm{kB} /$ p65 in HCSCs. Nuclear and cytoplasmic extracts were prepared from HCSCs (shBC047440) than in HCSCs (NC) and the expression of NF- $\mathrm{kB} / \mathrm{p} 65$ was determined by western blot analysis. (C) LSD tests by ANOVA were performed to determine the differences in the nuclear NF- $\mathrm{kB} / \mathrm{GAPDH}$, cytoplasmic $\mathrm{NF}-\mathrm{kB} / \mathrm{GAPDH}$ ratio between groups. ${ }^{*} \mathrm{P}<0.05$ compared to control group.
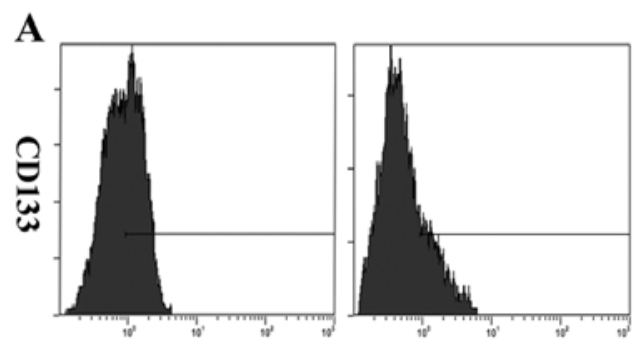

\section{B}
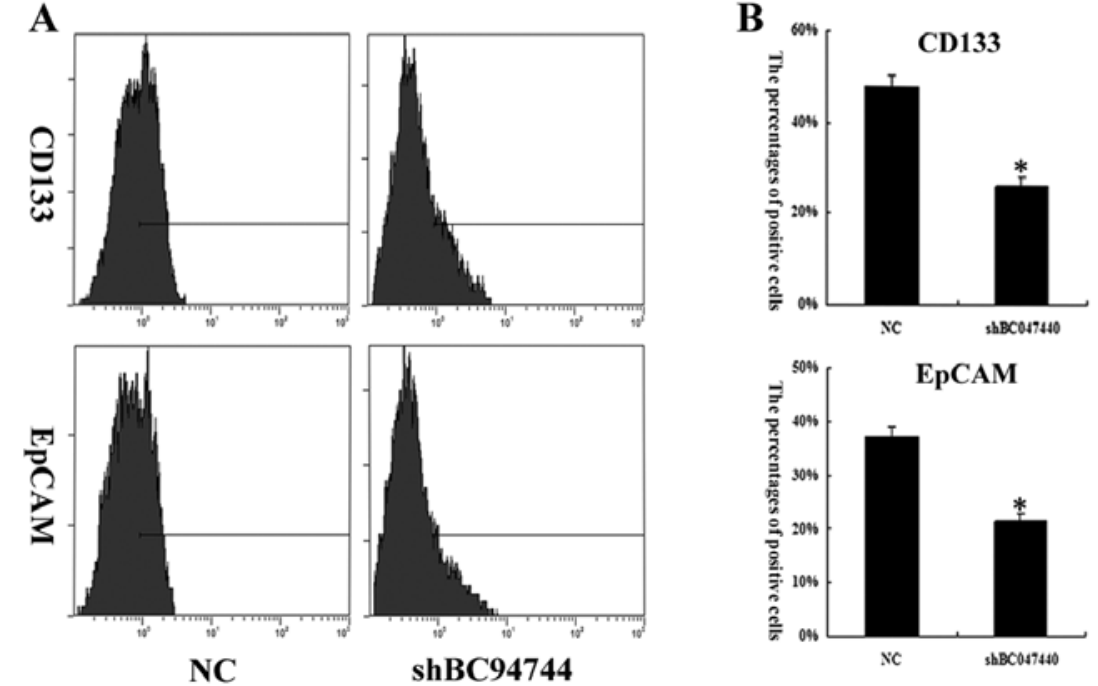

Figure 6. (A) On day 7 of cell growth in the presence of HGF, the expression of stem cell markers (CD133 and EpCAM) was different in HCSCs (shBC047440) and HCSCs (NC) by FACS. (B) The exact surface-molecule expression levels are shown in the column chart. "P<0.05 compared to control group.

HGF, HCSCs (shBC047440) produced significantly higher levels of urea compared to HCSCs (NC) (Fig. 7C).

To evaluate the fine structural characteristics of HCSCs (shBC047440) and HCSCs (NC), the cells cultured in the presence of HGF on day 7 were examined using TEM (Fig. 8).
HCSCs (shBC047440) exhibited morphological features of early differentiation similar to those of hepatocytes. Numerous mitochondria, lysosomes, prominent nucleoli, well-developed Golgi apparatuses, rough and smooth endoplasmic reticulum and especially glycogensomes (markers of mature hepatocytes) 

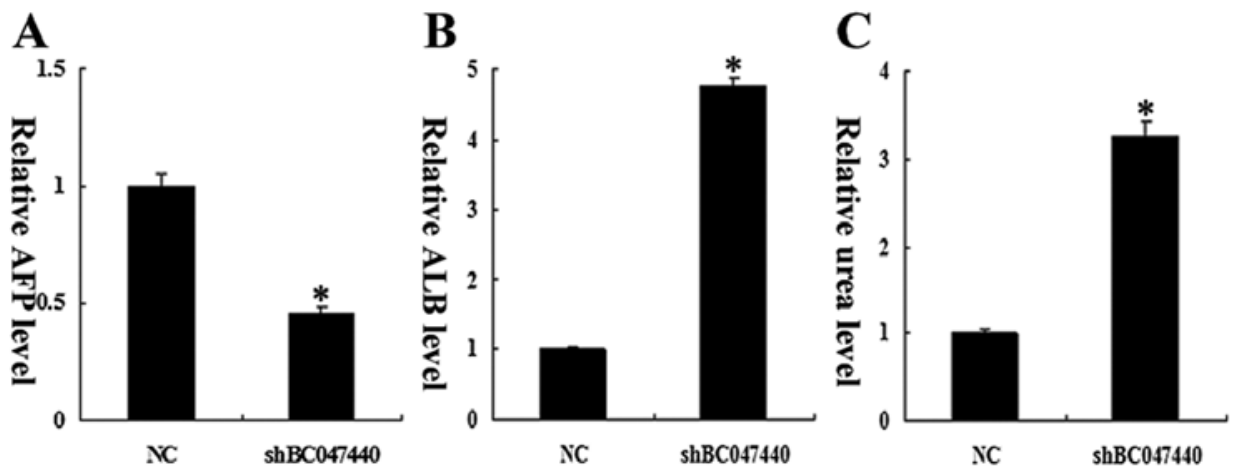

Figure 7. Functional tests of HCSCs (shBC047440) and HCSCs (NC) cultured in the presence of HGF for 7 days. (A) AFP, (B) ALB and (C) urea production by the HCSCs (shBC047440) and HCSCs (NC) was analyzed. The values of the HCSCs (NC) were set at $1 .{ }^{*} \mathrm{P}<0.05$ compared to control group.

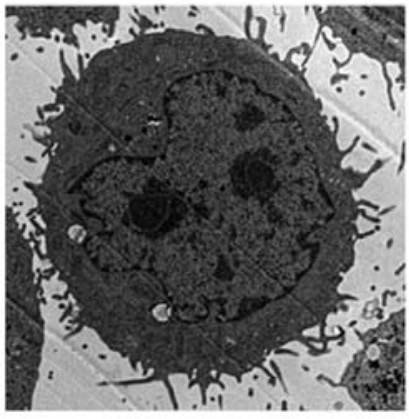

$\mathrm{NC}(\times 8000)$

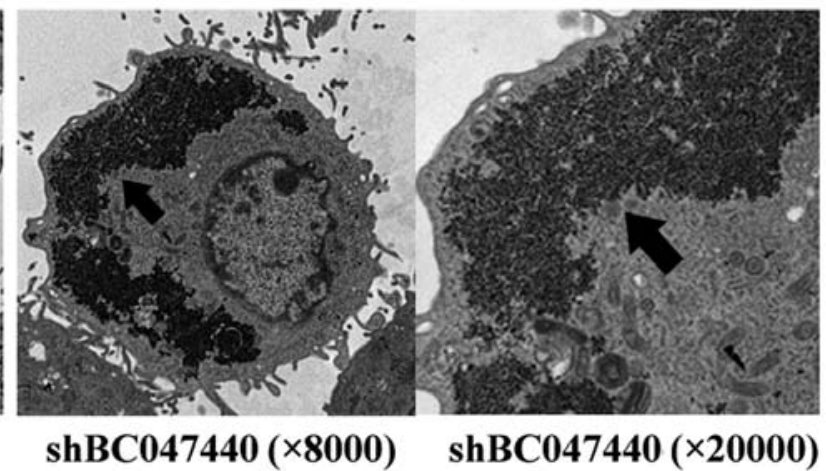

$\operatorname{shBC047440(\times 8000)} \quad \operatorname{shBC047440(\times 20000)}$

Figure 8. After 7 days in HGF culture, ultrastructural characteristics of HCSCs (shBC047440) and HCSCs (NC) were analyzed using TEM. Certain hepatocyte characteristics, including mitochondria, rough and smooth endoplasmic reticulum and glycogensome (black arrow), were observed in HCSCs (shBC047440) group.
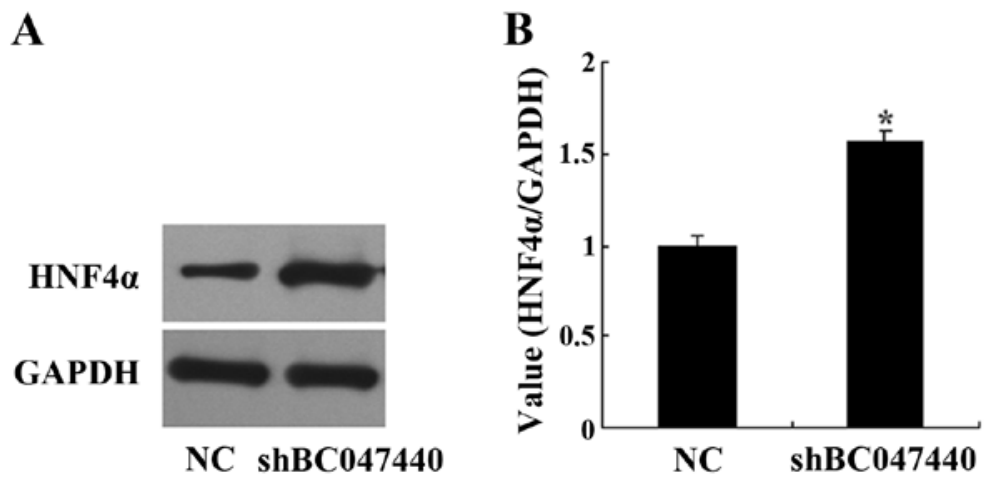

Figure 9. (A) Western blot analysis shows the effects of downregulated BC047440 on the expression of HNF4 $\alpha$ in HCSCs. (B) LSD tests by ANOVA were performed to determine the differences in the HNF4 /GAPDH ratio between groups. "P<0.05 compared to control group.

were observed in the cytoplasm of HCSCs (shBC047440). The nuclei displayed a low nuclear to cytoplasmic ratio and the cells were polarized, as shown by the presence of bile canaliculi. In contrast, the ultrastructural features of HCSCs (NC) were with large and regular nuclei, abundant ribosomes and mitochondria, poorly developed Golgi apparatus and endoplasmic reticulum, seldom lysosomes and high nuclear-cytoplasmic ratio. The images suggest that $\mathrm{BC} 047440$ can modulate HCSC differentiation and that suppression of BC047440 results in inducing HCSC differentiation into hepatocytes.
HNF4 $\alpha$ is important for hepatocyte development, differentiation and function. We investigated whether BC047440 knockdown alters the expression of HNF4 $\alpha$. As shown in Fig. 9, BC047440 knockdown induced an increase in $\mathrm{HNF} 4 \alpha$ protein levels.

BC047440 knockdown inhibits the tumorigenic capacity of HCSCs in vivo. To examine the effect of BC047440 depletion on cell tumorigenic capacity in vivo, xenograft tumor growth assays were performed in nude mice. Compared with 

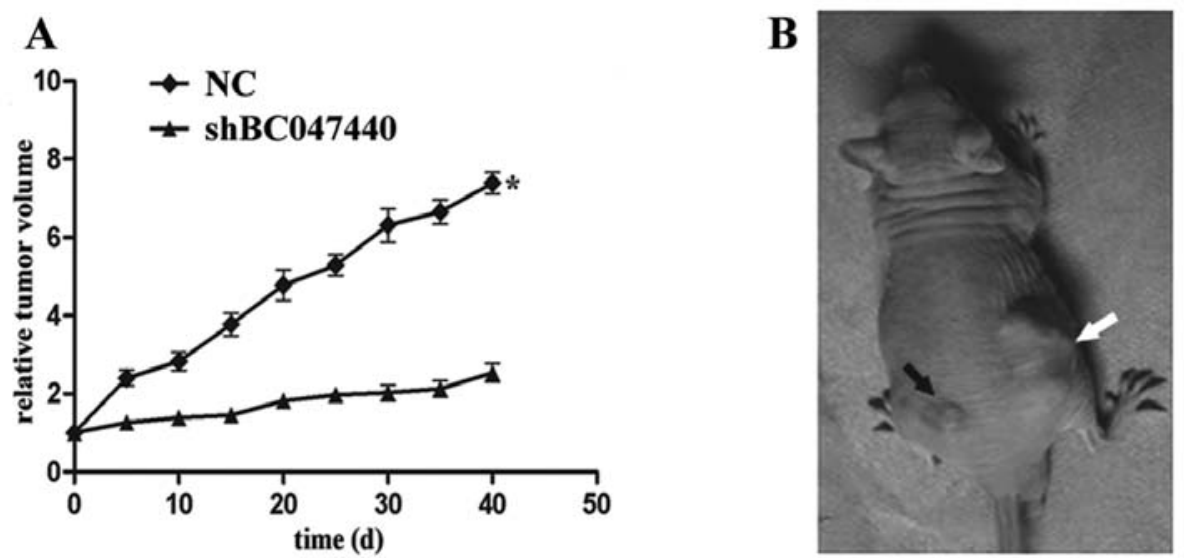

Figure 10. (A) Effect of BC047440 inhibition on tumorigenicity in nude mice of HCSCs. The tumor volumes were measured on the indicated day. ${ }^{*}<0.05$ vs. HCSC control group. (B) At 40 days after injection of BC047440-silent HCSCs and HCSCs respectively, representative tumors were observed. The arrows indicate HCSC (shBC047440) group (black) and HCSCs (NC) (white).

HCSCs, injection of BC047440-silent HCSCs led to markedly decreased tumor volume $(\mathrm{P}<0.05$; Fig. 10). Taken together, these data show that decreased expression of BC047440 in HCSCs effectively suppressed the tumorigenic capacity of HCSCs in vivo.

\section{Discussion}

HCSC-specific phenotypes and mechanisms that relate to functions in tumorigenicity, HCC progression, and therapeutic resistance have been identified. These results indicate that HCSCs may contribute to the failure of existing therapies to consistently eradicate malignant tumors (12-15). Therefore, HCSCs represent novel and translationally relevant targets for clinical cancer therapy. Notably, proof-of-principle experiments have strengthened the rationale for developing HCSC-targeted therapeutic modalities that might complement more conventional HCC therapies (16).

The malignant biological behavior of CSCs is mainly due to increased proliferation and immature state. Recent research showed that malignant behavior was most likely maintained and regulated by various genes and related pathways. For example, the Wnt pathway is closely related to CSC self-renewal and proliferation. Studies have shown that Wnt pathway activation plays an important role in maintaining the self-renewal and proliferation of leukemia stem cells and epithelium-derived CSCs (17). The activation of the Hedgehog pathway has been found in multiple myeloma and chronic myelogenous leukemia CSCs (18). In normal cells, p21 is an important regulator of the cell cycle. Studies of leukemia stem cells indicated that the expression of proto-oncogene PMLRAR upregulated p21 expression, which was closely related to leukemia stem cell proliferation (19). The proto-oncogene Bmil can promote selfrenewal and proliferation in normal cells, and recent studies reported that Bmil was overexpressed in the brain and in colonderived CSCs $(20,21)$. In addition, other factors such as Notch and human telomerase also play important roles in maintaining CSC function (22). The malignant behavior of CSCs and the complicated gene activation and related pathways that maintain CSC behavior can lead to drug resistance and the failure of traditional treatments. Therefore, we speculate that novel therapeutic approaches that target the genes and pathways responsible for maintaining CSC malignant behavior could improve cancer treatment. Fan et al (23) treated medulloblastoma cells with $\gamma$-secretase inhibitor to block Notch signaling and found that the proliferative ability of $\mathrm{CD} 133^{+}$cells was significantly reduced; however, the proliferative ability of CD133 cells was not affected. Verma et al (24) downregulated the expression of $\beta$-catenin, the key protein in the Wnt pathway in colon cancer cells, and found that the proliferative capacity of CSCs was effectively inhibited. Piccirillo et al (25) applied exogenous bone morphogenetic protein to induce glioblastoma stem cell differentiation and observed a significant reduction in the tumorigenic ability of the entire tumor cell population. The above studies suggested that manipulating the genes and related pathways responsible for maintaining CSC function could reduce proliferative capacity and induce CSC differentiation, improving the likelihood of achieving better treatment outcomes. Targeting CSCs is a novel and effective therapeutic strategy to inhibit tumor occurrence and growth. However, compared with the research on CSCs in solid tumors, HCSC research is still preliminary. There are relatively few studies regarding the effects of key genes and related pathways on HCSC malignant behavior. In addition, CSCs and normal stem cells share various pathways, such as Notch, Wnt and Bmi, and applying specific gene therapies targeting those pathways might raise safety concerns. Therefore, conducting in-depth research on the specific genes and pathways responsible for maintaining HCSC functions might lay a foundation for investigating the molecular mechanism of CSC pathogenesis, which will provide new CSC intervention targets.

The current studies regarding the roles of BC047440, a new HCC-related gene, in HCC were all completed by our research group, and the results have been widely recognized (7-9). In a previous investigation, we found that downregulated BC047440 expression can inhibit HepG2 cell proliferation, decreasing cellular AFP expression and significantly increasing functional gene expression in normal hepatocytes, such as ALB and cytochrome P450 1A2 (CYP1A2) (Fig. 1). The above results suggested that BC047440 might be an important molecule to mediate HCC proliferation and differentiation of HCC cells and might be useful for gene-targeted therapies. Recent studies 
confirmed that the existence of HCSCs was the main reason why traditional therapies designed to reduce tumor volume failed. How to inhibit HCSC functions in tumorigenesis and maintain $\mathrm{HCC}$ to achieve better treatment effects has become a hot topic. A growing body of evidence suggests that compared with traditional therapeutic approaches, HCSC-targeting therapy that aims to inhibit HCSC proliferation and promote tumor cell differentiation is a more effective way to eradicate $\mathrm{HCC}$ and reduce recurrence and metastasis rates. The development of HCSC-targeting therapy has always been an important research topic in our laboratory. However, it still faces many challenges, such as identifying the specific molecules involved in maintaining HCSC malignant behavior. At present, few such studies have been reported. Due to its important role in HCC, we considered whether BC 047440 could be one of the specific molecules involved in maintaining HCSC malignant behavior. To test the above hypothesis, we employed gene chips to screen for genes that are differentially expressed between HCSCs and HNSCs and found that BC047440 expression was significantly upregulated in HCSCs (data not shown). This result was subsequently confirmed with western blot analysis. Furthermore, we also applied an RNAi technique to interfere with BC047440 expression in HCSCs. Since the proliferative capacity of HCSCs and differentiation degree are closely related to their malignant behavior and given the important role of $\mathrm{BC} 047440$ in $\mathrm{HCC}$ proliferation and differentiation, we hypothesized that the absence of BC047440 might inhibit HCSC proliferation and promote HCSC differentiation. As we speculated, knockdown of BC047440 by shRNA in HCSCs leads to proliferation inhibition and differentiation induction in vitro and alters the expression of proliferation and differentiation-associated molecules. The in vivo experiment results indicated that inhibiting BC047440 expression significantly decreased HCSC tumorigenicity. Based on our study results, we described that $\mathrm{BC} 047440$ plays an important role in the proliferation and differentiation of HCSCs. However, further studies should be conducted to investigate this putative process in more detail.

By conducting studies on BC047440, we found that it might affect multiple proliferation- and differentiation-related proteins: i) NF- $\mathrm{NB}$ is a pleiotropic and multifunctional nuclear transcription factor involved in mediating tumor growth, proliferation, escaping apoptosis, invasion and metastasis (26). Studies have shown that $N F-\kappa B$ is activated in a variety of CSCs, and inhibiting $\mathrm{NF}-\kappa \mathrm{B}$ activation can inhibit CSC proliferation and induce apoptosis (27). We previously found that $\mathrm{BC} 047440$ can promote HepG2 proliferation by activating $\mathrm{NF}-\kappa \mathrm{B}$ signaling. After inhibiting BC047440 expression in HCSCs, the NF- $\kappa \mathrm{B}$ level was increased in the cytosol but decreased in the nucleus. Given the close relationship between $\mathrm{NF}-\kappa \mathrm{B}$ activation and CSC proliferation described above, as well as results from our studies, we considered $N F-\kappa B$ might play an important role in BC047440-regulated HCSC proliferation. However, $\mathrm{NF}-\kappa \mathrm{B}$ activation might not be the initiating factor of the proliferation signal pathway in HCSCs. Rather, it might be a key factor in the signaling pathway to promote cell proliferation, and other genetic products might be involved. For example, the essential upstream molecules of NF- $\kappa \mathrm{B}$, such as TRAF2, TRAF5, TRAF6, TRAF1, RIP, TLR, Myd88, IRAK1, IRAK2, TAK1 and TAK2, can interact with $\mathrm{NF}-\kappa \mathrm{B}$ to maintain its sustained activity and promote HCSC proliferation. However, which gene products play roles in the $\mathrm{NF}-\kappa \mathrm{B}$ signaling pathway are unknown, as are the specific mechanisms of those gene products. At present, our understanding regarding the regulation of $\mathrm{BC} 047440 / \mathrm{NF}-\kappa \mathrm{B}$ signaling in HCSC proliferation remains preliminary. ii) $\mathrm{HNF} 4 \alpha$ is a member of the nuclear receptor superfamily and is highly expressed in mature hepatocytes. It is an important transcription factor that regulates hepatocyte development, differentiation and function. It plays a critical role in liver development, as well as gene transcription and regulation in adult hepatocytes. In addition, HNF4 $\alpha$ overexpression can facilitate tumor cell transformation into lower degrees of malignancy and reverse the de-differentiated stage of HCC cells (28). We examined the expression level of HNF4 $\alpha$ in preliminary experiments and found that the absence of $\mathrm{BC} 047440$ expression was accompanied by significantly elevated HNF4 $\alpha$ expression in HepG 2 cells, suggesting that $\mathrm{BC} 047440$ can promote HepG2 differentiation through HNF4 $\alpha$ signaling (Fig. 1). Inhibiting BC047440 expression in HCSCs leads to significantly elevated HNF4 $\alpha$ expression. In-depth studies on whether BC047440 can regulate HCSC differentiation through $\mathrm{HNF} 4 \alpha$ signaling will help to elucidate the mechanism of tumorigenesis in HCC and provide important experimental evidence for the application of gene-targeted therapy in HCC treatment. iii) BC047440 dysregulation can lead to transcriptional activation of downstream proteins that play important roles in HCSC proliferation and differentiation. However, the specific functions of induced gene expression are not fully understood. The identification of specific downstream target molecules in the BC047440 signaling pathway requires further research.

We preliminarily confirmed the novel HCC-related gene BC047440 as an important molecule that induces malignant behavior in HCSCs. The absence of BC047440 may be associated with reduced HCSC proliferation and increased differentiation. However, elucidating the specific mode of action requires further studies. Furthermore, the precise molecular mechanism of BC04744's involvement in maintaining HCSC malignant behavior remains unclear. The answers to these questions will provide a foundation to elucidate the function and molecular mechanism of BC047440 during HCC onset and development, which can provide theoretical and practical evidence for developing a novel gene target for HCC therapy.

\section{Acknowledgements}

The authors thank Juan Li for her excellent technical assistance. The present study was funded by the Chinese National Natural Science Foundation (grant no. 81372561, 81302168) and the Natural Science Foundation of Chongqing (grant no. cstc2012jjA10079).

\section{References}

1. Shiozawa Y, Nie B, Pienta KJ, Morgan TM and Taichman RS: Cancer stem cells and their role in metastasis. Pharmacol Ther 138: 285-293, 2013.

2. Cheng $X$ and $\mathrm{O}^{\prime}$ Neill HC: Oncogenesis and cancer stem cells: current opinions and future directions. J Cell Mol Med 13: 4377-4384, 2009. 
3. Yamashita T and Wang XW: Cancer stem cells in the development of liver cancer. J Clin Invest 123: 1911-1918, 2013.

4. Padhya KT, Marrero JA and Singal AG: Recent advances in the treatment of hepatocellular carcinoma. Curr Opin Gastroenterol 29: 285-292, 2013

5. Wilson GS, Hu Z, Duan W, et al: Efficacy of using cancer stem cell markers in isolating and characterizing liver cancer stem cells. Stem Cells Dev 22: 2655-2664, 2013.

6. Lee H, Kim JB, Park SY, Kim SS and Kim H: Combination effect of paclitaxel and hyaluronic acid on cancer stem-like side population cells. J Biomed Nanotechnol 9: 299-302, 2013.

7. Zheng L, Liang P, Li J, et al: ShRNA-targeted COMMD7 suppresses hepatocellular carcinoma growth. PLoS One 7: e45412, 2012.

8. Zheng L, Huang X, Liang P, et al: BC047440 overexpression is a risk factor for tumor invasion and poor prognosis in hepatocellular carcinoma. Hepatogastroenterology 57: 919-925, 2010.

9. Zheng L, Liang P, Zhou J, Huang X, Wen Y, Wang Z and Li J: BC047440 antisense eukaryotic expression vectors inhibited HepG2 cell proliferation and suppressed xenograft tumorigenicity. Braz J Med Biol Res 45: 97-103, 2012.

10. Liu WH, Li R and Dou KF: Convenient and efficient enrichment of the $\mathrm{CD} 133^{+}$liver cells from rat fetal liver cells as a source of liver stem/progenitor cells. Stem Cell Rev 7: 94-102, 2011.

11. Liu WH, Wang X, You N, et al: Efficient enrichment of hepatic cancer stem-like cells from a primary rat HCC model via a density gradient centrifugation-centered method. PLoS One 7: e35720, 2012.

12. Li CH, Wang YJ, Dong W, et al: Hepatic oval cell lines generate hepatocellular carcinoma following transfection with $\mathrm{HBx}$ gene and treatment with aflatoxin B1 in vivo. Cancer Lett 311: 1-10, 2011.

13. Chiba T, Kita K, Zheng YW, et al: Side population purified from hepatocellular carcinoma cells harbors cancer stem cell-like properties. Hepatology 44: 240-245, 2006.

14. Zhu Z, Hao X, Yan M, Yao M, Ge C, Gu J and Li J: Cancer stem/ progenitor cells are highly enriched in $\mathrm{CD} 133^{+} \mathrm{CD} 44^{+}$population in hepatocellular carcinoma. Int J Cancer 126: 2067-2078, 2010.

15. Espandiari P, Robertson LW, Srinivasan C and Glauert HP: Comparison of different initiation protocols in the resistant hepatocyte model. Toxicology 206: 373-381, 2005.
16. Yamashita T, Honda M, Nakamoto Y, et al: Discrete nature of $\mathrm{EpCAM}^{+}$and $\mathrm{CD} 90^{+}$cancer stem cells in human hepatocellular carcinoma. Hepatology 57: 1484-1497, 2013.

17. Malanchi I, Peinado H, Kassen D, et al: Cutaneous cancer stem cell maintenance is dependent on $\beta$-catenin signalling. Nature 452: 650-653, 2008.

18. Zhao $\mathrm{C}$, Chen A, Jamieson $\mathrm{CH}$, et al: Hedgehog signalling is essential for maintenance of cancer stem cells in myeloid leukaemia. Nature 458: 776-779, 2009.

19. Viale A, De Franco F, Orleth A, et al: Cell-cycle restriction limits DNA damage and maintains self-renewal of leukaemia stem cells. Nature 457: 51-56, 2009.

20. Cui H, Hu B, Li T, Ma J, Alam G, Gunning WT and Ding HF: Bmi-1 is essential for the tumorigenicity of neuroblastoma cells. Am J Pathol 170: 1370-1378, 2007.

21. Kim JH, Yoon SY, Kim CN, et al: The Bmi-1 oncoprotein is overexpressed in human colorectal cancer and correlates with the reduced p16INK4a/p14ARF proteins. Cancer Lett 203: 217-224, 2004.

22. Song $\mathrm{K}$, Wu $\mathrm{J}$ and Jiang C: Dysregulation of signaling pathways and putative biomarkers in liver cancer stem cells (Review). Oncol Rep 29: 3-12, 2013.

23. Fan X, Matsui W, Khaki L, Stearns D, Chun J, Li YM and Eberhart CG: Notch pathway inhibition depletes stem-like cells and blocks engraftment in embryonal brain tumors. Cancer Res 66: 7445-7452, 2006

24. Verma UN, Surabhi RM, Schmaltieg A, Becerra C and Gaynor RB: Small interfering RNAs directed against $\beta$-catenin inhibit the in vitro and in vivo growth of colon cancer cells. Clin Cancer Res 9: 1291-1300, 2003.

25. Piccirillo SG, Reynolds BA, Zanetti N, et al: Bone morphogenetic proteins inhibit the tumorigenic potential of human brain tumour-initiating cells. Nature 444: 761-765, 2006.

26. DiDonato JA, Mercurio F and Karin M: NF- $\mathrm{BB}$ and the link between inflammation and cancer. Immunol Rev 246: 379-400, 2012.

27. Prud'homme GJ: Cancer stem cells and novel targets for antitumor strategies. Curr Pharm Des 18: 2838-2849, 2012.

28. Ning BF, Ding J, Yin C, et al: Hepatocyte nuclear factor $4 \alpha$ suppresses the development of hepatocellular carcinoma. Cancer Res 70: 7640-7651, 2010. 